\title{
BMJ Open PATHway I: design and rationale for the investigation of the feasibility, clinical effectiveness and cost-effectiveness of a technology-enabled cardiac rehabilitation platform
}

\author{
Jomme Claes, ${ }^{1}$ Roselien Buys, ${ }^{1}$ Catherine Woods, ${ }^{2}$ Andrew Briggs, ${ }^{3}$ \\ Claudia Geue, ${ }^{3}$ Moira Aitken, ${ }^{3}$ Niall Moyna, ${ }^{4}$ Kieran Moran, ${ }^{4}$ Noel McCaffrey, ${ }^{4}$ \\ loanna Chouvarda, ${ }^{5}$ Deirdre Walsh, ${ }^{4}$ Werner Budts, ${ }^{1,6}$ Dimitris Filos, ${ }^{5}$ \\ Andreas Triantafyllidis, ${ }^{5}$ Nicos Maglaveras, ${ }^{5}$ Véronique A Cornelissen ${ }^{7}$
}

To cite: Claes J, Buys R, Woods C, et al. PATHway I: design and rationale for the investigation of the feasibility, clinical effectiveness and costeffectiveness of a technologyenabled cardiac rehabilitation platform. BMJ Open 2017;7:e016781. doi:10.1136/ bmjopen-2017-016781

- Prepublication history for this paper is available online. To view these files please visit the journal online (http://dx.doi. org/10.1136/bmjopen-2017016781)

Received 9 March 2017

Revised 16 May 2017

Accepted 16 May 2017



For numbered affiliations see end of article.

Correspondence to Dr Roselien Buys; roselien. buys@faber.kuleuven.be

\section{ABSTRACT}

Introduction Exercise-based cardiac rehabilitation (CR) independently alters the clinical course of cardiovascular diseases resulting in a significant reduction in all-cause and cardiac mortality. However, only $15 \%-30 \%$ of all eligible patients participate in a phase 2 ambulatory programme. The uptake rate of community-based programmes following phase $2 \mathrm{CR}$ and adherence to long-term exercise is extremely poor. Newer care models, involving telerehabilitation programmes that are delivered remotely, show considerable promise for increasing adherence. In this view, the PATHway (Physical Activity Towards Health) platform was developed and now needs to be evaluated in terms of its feasibility and clinical efficacy. Methods and analysis In a multicentre randomised controlled pilot trial, 120 participants $(\mathrm{m} / \mathrm{f}$, age 40 80 years) completing a phase 2 ambulatory CR programme will be randomised on a 1:1 basis to PATHway or usual care. PATHway involves a comprehensive, internetenabled, sensor-based home CR platform and provides individualised heart rate monitored exercise programmes (exerclasses and exergames) as the basis on which to provide a personalised lifestyle intervention programme. The control group will receive usual care. Study outcomes will be assessed at baseline, 3 months and 6 months after completion of phase 2 of the CR programme. The primary outcome is the change in active energy expenditure. Secondary outcomes include cardiopulmonary endurance capacity, muscle strength, body composition, cardiovascular risk factors, peripheral endothelial vascular function, patient satisfaction, health-related quality of life (HRQoL), well-being, mediators of behaviour change and safety. HRQoL and healthcare costs will be taken into account in cost-effectiveness evaluation.

Ethics and dissemination The study will be conducted in accordance with the Declaration of Helsinki. This protocol has been approved by the director and clinical director of the PATHway study and by the ethical committee of each participating site. Results will be disseminated via peer-reviewed scientific journals and presentations at congresses and events.

\section{Strengths and limitations of this study}

- PATHway (Physical Activity Towards Health) provides comprehensive cardiac rehabilitation in the living room of the patient.

- The telerehabilitation intervention is fully personalised on the patients' needs, goals and preferences.

- This pilot study could justify prospective trials to evaluate the longer-term effects of the PATHway platform on cardiovascular health.

- The study is of relatively short duration and no data will be available after 6 months.

- The study is underpowered to detect statistically significant changes for a majority of secondary outcomes.

Trial registration number NCT02717806. This trial is currently in the pre-results stage.

\section{INTRODUCTION}

Cardiovascular diseases (CVD) are the major reason for premature death and disability in Europe, resulting in approximately 4.3 million deaths and costing the European Union $€ 196$ billion per year. ${ }^{1-3}$ Studies over the last decades have shown an increasing prevalence and incidence of CVD. Incidence increased by $5 \%$ from 1990 to 2000 and an additional increase of $25 \%$ is expected by 2030. ${ }^{13}$ With these expected increasing numbers of patients eligible for cardiac rehabilitation (CR), the importance of CR will only grow.

Exercise-based CR favourably and independently alters the clinical course of CVD with a 15\%-31\% reduction in all-cause and cardiac mortality. ${ }^{4}$ Peak aerobic capacity 
appears to be a strong predictor of mortality in patients with CVD. ${ }^{6-8}$ Hence, the impetus to improve exercise capacity in patients with CVD in the short term and maintain this improvement in the longer-term is strong. There is overwhelming evidence coming from meta-analytic research showing that exercise-based CR programmes are able to significantly increase this exercise capacity by 20\%-30\%. ${ }^{9}$ Based on such accumulating evidence, physical activity counselling and exercise training are now core components in all secondary prevention guidelines for patients with cardiac disease. ${ }^{10}$

However, only $15 \%-30 \%$ of all patients eligible for CR engage in a formal exercise programme (phase 2 ambulatory programme). ${ }^{11}$ Moreover, uptake rates of community-based CR following these phase 2 programmes are even lower and the number of patients effectively continuing the long-term rehabilitation phase is extremely poor. ${ }^{11}$ Optimising CR delivery and improving the ongoing adoption of a healthy lifestyle will be important for the health and well-being of the general population and from the perspective of healthcare costs. The main reasons for the low adherence rates include: travel distance, low self-efficacy, perceived bodyimage and lack of time. ${ }^{12}$ Therefore, it is a priority to find effective interventions that result in a lifelong physically active lifestyle. Ideally, such interventions should lead to a sustainable cardioprotective lifestyle resulting in an increase of moderate to vigorous physical activity and an improved physical fitness, enhancing both survival and health-related quality of life (HRQoL) at a cost that represents good value for money for health systems.

An evidence-based approach to inducing sustained lifestyle changes involves the implementation of health behaviour change strategies grounded in health behaviour change theory. ${ }^{13}$ A range of health behaviour change techniques can be used to enhance intervention effectiveness. ${ }^{14}$ Frequent feedback and the provision of motivation and support have been shown to be efficacious. ${ }^{1516}$ Setting realistic goals and individualising the programme to the needs and interests of the patient improves compliance to physical activity and exercise. ${ }^{17}$ To this end, state-ofthe-art telerehabilitation ${ }^{18}$ technology has the potential to help participants by inducing sustained lifestyle changes and health providers by providing personalised health services. ${ }^{19}$ Patients with CVD show high interest in technology enabled CR, providing a clear indication to develop such interventions. ${ }^{20}$

PATHway (Physical Activity Towards Health) is a novel approach set within a collaborative care context with health professionals that aims to empower participants to self-manage their CVD risk factors. This will be achieved using a patient-centric holistic behavioural change approach that involves an internet-enabled and sensorbased home exercise platform as the core component of a personalised, comprehensive lifestyle intervention programme. This will enable participants to both better understand and deal with their own condition and adopt and/or maintain a healthy lifestyle in general.

\section{AIMS AND OBJECTIVES}

The study will use a single blind multicentre pilot randomised controlled clinical trial to assess the impact of the PATHway platform on physical activity, lifestyle and health-related physical fitness in patients with CVD and to evaluate the acceptability, feasibility and cost-effectiveness of using PATHway in patients with CVD.

\section{Primary objective of the trial}

The primary objective is to compare objectively measured active energy expenditure. We hypothesise that physical activity defined as the total weekly volume of active (activities requiring an energy expenditure $>3$ metabolic units) energy expenditure (EE in kcal) will be significantly greater in the intervention (PATHway) than the control group (usual care) at 6 months.

\section{Secondary objectives of the trial}

- Compare cardiopulmonary endurance capacity, muscle strength and muscle endurance, flexibility, cardiovascular risk score and body composition.

- Compare psychosocial well-being and quality of life including depression, self-efficacy and social relationship.

- Participants are much more likely to adhere to PATHway if they understand the system and like how it operates. Through process evaluation the feasibility, acceptability, satisfaction and usability of the PATHway system from the participants' perspective will be assessed.

- Explore the determinants/moderators of health behaviour change and CV health.

- Estimate the cost-effectiveness of PATHway.

- Assess the safety of PATHway.

- Evaluate whether the decision support system (DSS) of PATHway ensures the safety of the participants, optimises the exercise programme according to patient needs and contributes to health behaviour change.

\section{METHODS}

The PATHway study protocol is reported following the Standard Protocol Items: recommendations for Interventional Trials (SPIRIT) $2013,{ }^{21} 22$ the checklist of which can be found as the added Research Checklist. This trial is registered at ClinicalTrials.gov: NCT02717806.

\section{Study design}

A single blind, parallel two-group pilot multicentre randomised controlled design will be employed. A total of 120 participants will be randomised to the PATHway intervention or usual care. Given the nature of the intervention, it is not possible to blind the participants or the researchers implementing PATHway. Researchers performing the outcome assessments will, however, be blinded to patient allocation. Figure 1 shows the study flow. 


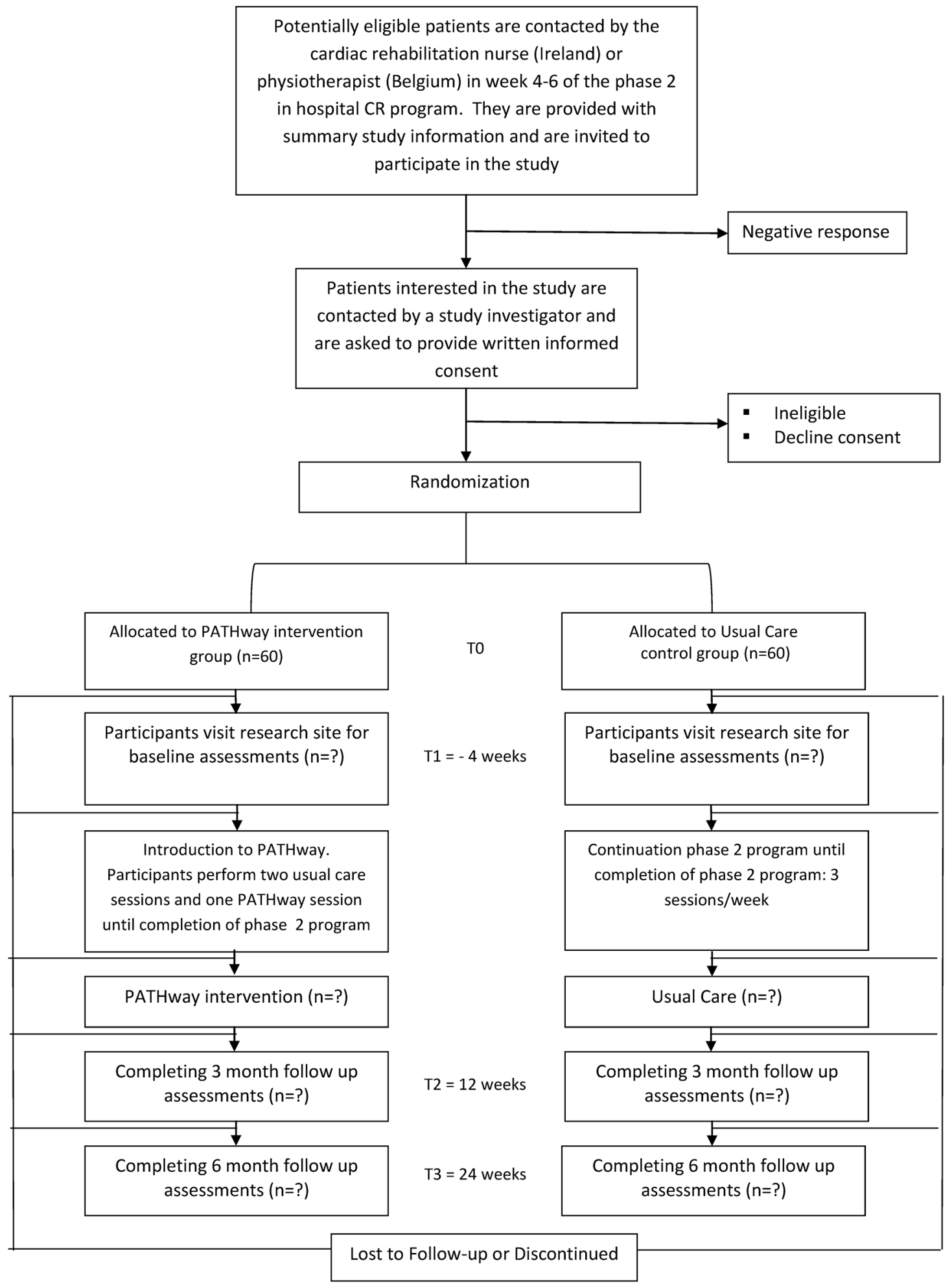

Figure 1 Study schedule. CR, cardiac rehabilitation; PATHway, Physical Activity Towards Health.

\section{Study setting}

Participants will be recruited among patients with cardiac disease participating in three outpatient CR programmes in Belgium and Ireland. Sixty participants will be recruited in the University Hospitals Leuven (Belgium), 30 participants will be recruited from the community clinic at Beaumont Hospital, Dublin (Ireland) and another 30 participants will be recruited from the community clinic at The Mater
Misericordiae University Hospital, (Ireland). The University of Leuven will be the coordinating centre for the trial. Daily management of the study will be performed by a local principal investigator at each participating site.

\section{Eligibility criteria and recruitment}

The study population will comprise 120 patients diagnosed with CVD. Men and women between the ages of 
Table 1 Inclusion and exclusion criteria for the PATHway (Physical Activity Towards Health) trial

\begin{tabular}{lc}
\hline Inclusion criteria & Exclusion criteria \\
\hline Men and women with documented cardiovascular disease & Significant illness during the last 6 weeks \\
who are enrolled for the first time in a CR programme & Known severe ventricular arrhythmia with functional or \\
Aged 40-80years & prognostic significance \\
Participants are on optimal medical treatment and stable & Significant myocardial ischaemia, haemodynamic \\
with regard to symptoms and pharmacotherapy for at least & deterioration or exercise-induced arrhythmia at baseline \\
4 weeks & testing \\
Participants must complete the ambulatory CR programme & Cardiac disease that limits exercise tolerance (valve disease \\
and receive clinical approval from their treating physician to & with significant haemodynamic consequences, hypertrophic \\
continue exercising outside the hospital programme & cardiomyopathy and so on) \\
Internet access at home & Comorbidity that may significantly influence 1-year \\
Sufficient space to deploy and use the system adequately & prognosis \\
& Functional or mental disability that may limit exercise \\
& Acute or chronic inflammatory diseases or malignancy, the \\
& use of anti-inflammatory drugs or immune suppression \\
& Severe chronic obstructive pulmonary disease (FEV1<50\%) \\
& NYHA class 4
\end{tabular}

$\mathrm{CR}$, cardiac rehabilitation.

40-80 years with documented CVD, who enrol for the first time in an outpatient CR programme, will be recruited during a 1-year period starting in May 2017. Participants entering the CR programme will be assessed for eligibility by the local hospital cardiac nurse (Ireland) or physiotherapist (Belgium). A detailed description of the inclusion and exclusion criteria is shown in table 1. Identified eligible participants will be contacted by a member of the research team at DCU and KU Leuven and provided with a full oral explanation of the design and purpose of the study, responsibilities of the participants, reasonable foreseeable inconveniences, confidentiality of the information collected. Patients who agree to participate will provide contact details and basic sociodemographic data.

\section{Randomisation and concealed allocation}

After signing an informed consent, participants will be randomised to either the PATHway-intervention or the usual care group. Randomisation will be performed in a 1:1 ratio, stratified by centre of enrolment. Randomisation schedules will be generated for each centre using a computerised random number generator. In order to assure concealment of allocation and minimise selection bias, an independent designated member of the coordinating centre in KU Leuven will assign a randomisation number in response to a request from a local investigator. Participants will receive a personalised identification code (PIC) which will be used on all subsequent transcripts and data collected to assure anonymity of all data.

\section{Sample size calculation}

As this is the first study with this type of complex intervention, without precedence on which to base accurate power calculations, a power calculation has not been performed. A major aim of the study is to provide evidence showing an increased uptake/compliance to a short-term technology-based physically active lifestyle intervention compared with usual care. It is envisaged that the study findings will provide data for the design of a larger randomised trial examining a technology-based physically active lifestyle intervention compared with usual care. We have chosen a convenience sample of 120 participants, in order to assess the plausible effects of short-term technology-based physically active lifestyle intervention on physical activity uptake as well as to provide an indication of the effect size on health-related fitness which we envisage would be the primary outcome of a future larger trial in patients with CVD.

\section{PATHway intervention}

As shown in figure 1, eligible and consenting participants will be enrolled prior to discharge from an outpatient ambulatory CR programme. In addition to the 2-3 weekly exercise sessions as part of the outpatient CR programme, participants allocated to the PATHway intervention will also undertake a single supervised familiarisation session during each of the last 4 weeks in order to systematically learn and practice all features of the platform. During this familiarisation period, a local investigator will install the PATHway system in the participant's home. Participants will be provided with a standardised training manual and set-up guide. During the familiarisation period, they will be requested to interact with the PATHway system by means of standardised assignments to assure they get acquainted with its features. Subsequently, participants will be encouraged to discuss their experience with the PATHway technology and identify problems.

The home-based PATHway system will provide an individualised exercise prescription based on the results of 


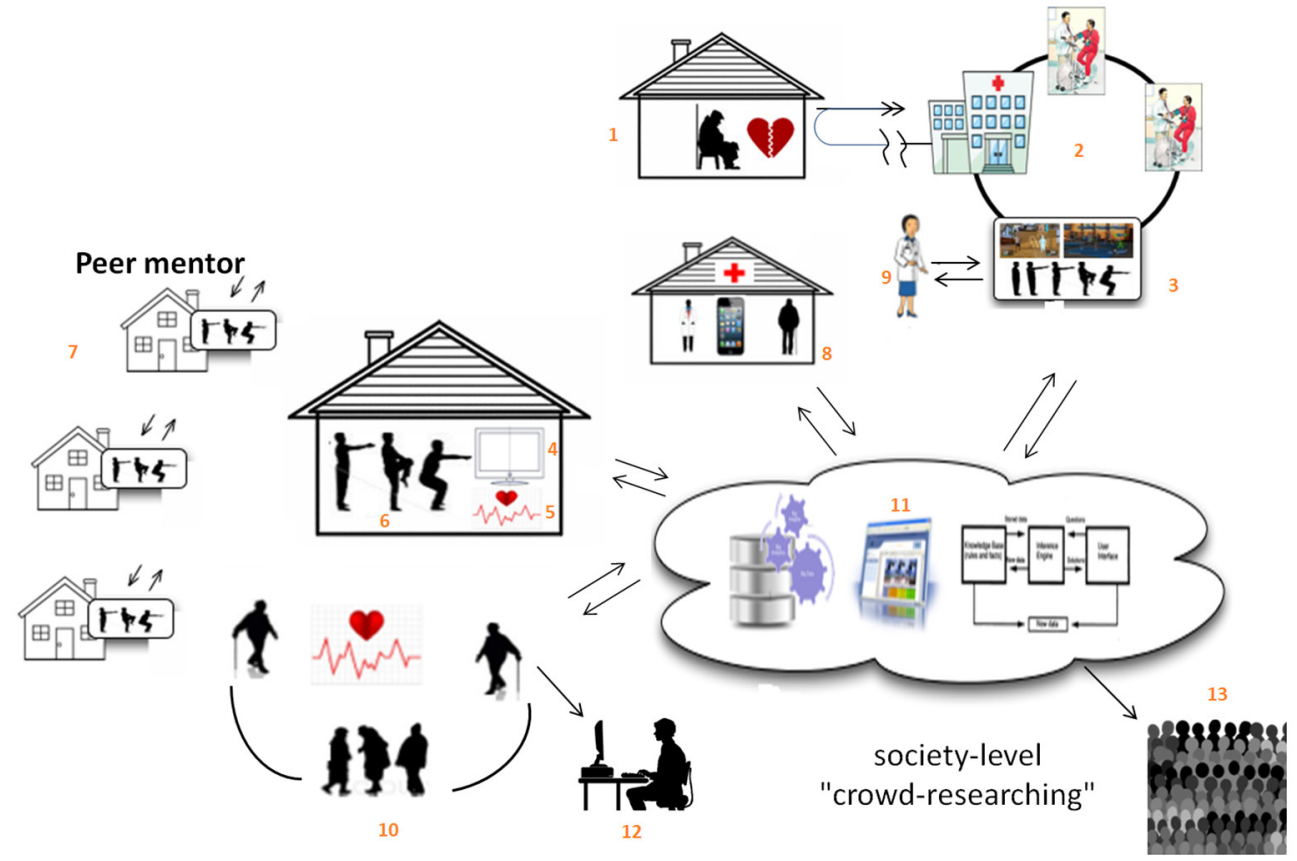

Figure 2 An overview of the PATHway (Physical Activity Towards Health) components: 1, the patient experiences a cardiac event; 2 , the patient is treated in the hospital; 3 , the patient participates in a phase 2 cardiac rehabilitation (CR) programme; $4+5+6$, the patient uses PATHway after completion of phase 2 CR; 7 , the patient is able to socially interact with other PATHway users via the system; 8, patient data are transmitted and used as feedback for the patient and health professionals; 9, this can allow an improvement of care by the health professional; $10+12$, the patient can also monitor outdoor activities and upload them to the system afterwards; 11, patient data are aggregated and allow for possibility of undertaking large-scale, accurate, longitudinal health-based research - 'crowd sourced researching' (13).

a cardiopulmonary exercise test performed at the start of the familiarisation period and according to current guidelines. ${ }^{1}$ 23-30 Additionally, a behavioural change component that allows realistic goal setting by the patient will be implemented with regard to diet, smoking cessation, alcohol, stress reduction and medication adherence. Guiding the patient to choose appropriate goals that focus on specific achievable health behaviour changes has been shown previously to promote goal attainment. ${ }^{13}$ Patients will be asked to complete the behaviour change assessment module during this familiarisation phase. Following this assessment, patients will be asked to choose a lifestyle behaviour they want to improve.

As shown in figure 2, the PATHway intervention is a comprehensive and complex self-care CR system. It involves the implementation of an end-to-end modular technology platform (ie, portable PC including PATHway software, Microsoft Kinect camera, Microsoft Band 2 heart rate monitor, Blood pressure device, Zensor 3-lead ECG device) that allows participants to better self-manage their illness. PATHway consists of five different modules; exercise, outdoor physical activity, behavioural change, social connectivity and affective response.

The home-based exercise module comprises an exercise class (exerclass) and an exercise game (exergame) component. The exerclass includes dynamic aerobic exercises, dynamic resistance exercises and relaxation. Participant movements, repetition count, energy expenditure and heart rate (HR) will be continuously monitored by the Microsoft Band $2^{31}$ in order to provide personalised feedback via a virtual 'avatar' coach and to continuously adapt the programme. A motion-capturing component using the Microsoft Kinect sensor will assess participants' movements during the exerclass and exergame sessions and provide them with feedback on the accuracy of their exercise execution. A DSS using HR (Microsoft Band 2) ${ }^{31}$ along with the accuracy derived from the motion capturing component will be used to dynamically select exercises during real time. ${ }^{32}$ In this context, the goal is to adjust ${ }^{32}$ the exercise prescription to achieve the prescribed target HR zone ${ }^{33}$ required to maintain and/or improve cardiovascular fitness. Finally, a prescreening data processing component will evaluate the participants resting HR and blood pressure, medication compliance and eating behaviour prior to starting each exercise session.

The exercise module will be supplemented by an outdoor physical activity module. A wrist worn HR/physical activity monitor (Microsoft band 2) will assess sitting behaviour and physical activity levels beyond the exerclasses and exergames. The combined data derived from the exercise module and outdoor physical activity assessment module will be aggregated to generate a physical activity report. The report will allow participants to monitor their physical activity behaviour and will provide the healthcare professionals with objective data relating to physical activity, sedentary behaviour and compliance. 
Substantial evidence indicates that telerehabilitation interventions are more effective when they incorporate a behaviour change module such as self-monitoring, providing feedback on performance, goal-setting and rewards. ${ }^{34}$ Behaviour change techniques based on the taxonomy developed by Michie and colleagues ${ }^{14}$ will be implemented in the PATHway platform within a behavioural change module. These will be delivered implicitly and according to patient preferences throughout the system as well as via the avatar, text-based resource content and push notifications linked with performance feedback. ${ }^{35}$

To further facilitate social support within a community context, a social connectivity module will enable small groups of remote participants to exercise together by allowing them to communicate during the exercise session by means of headsets, as well as by sending messages and a live chat function. ${ }^{36}$ An online calendar will also allow participants to promote events and to invite others to join, with a maximum of four participants per event.

The affective response module will use a questionnaire to measure the extent to which the participants enjoy each exercise session. The perceptions of effort, strain, discomfort or fatigue experienced during exercise will be measured using the Borg 16 point rating of perceived exertion (RPE) scale. The effect and RPE scores will be used to allow the PATHway platform to automatically adjust the exercise sessions offline according to the participant's levels of enjoyment and exertion.

An incorporated e-learning platform involving text, photo and video messaging information will provide participants with information on reducing lifestyle-related risk factors for CVD. A detailed overview of the PATHway system can be found elsewhere. ${ }^{37}$

Patients randomised to the PATHway intervention will be asked to use the system for 6 months following their outpatient CR programme.

\section{Usual care or control group}

During the 4-week familiarisation period, participants allocated to the usual care group will continue to attend the hospital-based outpatient CR programme 3 days per week. At completion of the outpatient CR programme, they will receive advice on how best to maintain a physically active lifestyle. They will be free to participate in any type of fitness classes, walking groups, cycling groups, available community-based CR programmes and so on. However, they will not be provided with a specific exercise prescription or receive direct feedback or support with regard to their physical activity behaviour during the 6-month follow-up period.

\section{Concomitant care}

Both intervention and control group will continue to receive medical and pharmacological management as well as the standard lifestyle advice according to prevailing guidelines. ${ }^{38} 39$

\section{Outcome measures and data collection}

Outcome measures will be assessed by researchers blinded to the group allocation. All assessments will be performed at baseline (t1), which coincides with the start of the familiarisation phase and at 3 months (t2) and 6 months (t3) after completion of the phase 2 CR programme unless otherwise specified (figure 1). Table 2 gives a tabulated summary of the study schedule and measurements.

\section{Primary outcome}

The primary outcome, total volume of weekly active energy expenditure (>3METs), will be objectively measured by means of a validated physical activity device, the ActiGraph GT9X Link (ActiGraph, Pensacola, Florida, USA). Measurements of physical activity and exercise capacity ${ }^{40}$ will be performed in the week before the PATHway run-in period. Both measurements will be repeated during the last week of both the third month $(=\mathrm{t} 2)$ and the sixth month (=t3) following completion of the phase 2 CR period.

\section{Secondary outcomes}

Health-related outcomes

At each data collection time point, participants will attend for a single visit between 09:00 and 12:00 hours following a 12-hour fast. After measurements of anthropometrics, vascular health and blood sampling have been performed, they will be given the time to consume a light breakfast. Following this, measurements on flexibility and muscle function will be performed. All measurements will be performed by the same blinded researchers on each participating site. In the following order:

- Body composition: weight, height, fat percentage, waist and hip circumferences. ${ }^{41}$

- Vascular function will be assessed by measuring the percentage change in brachial artery flow mediated dilation following $5 \mathrm{~min}$ of forearm occlusion. ${ }^{42} 43$ Blood pressure will be measured at rest (office BP) in sitting position.

- A 12-hour fasting blood sample will be drawn to measure selected biomarkers: fasting glucose, insulin, total cholesterol, high-density lipoprotein cholesterol, low-density lipoprotein cholesterol, triglycerides and HbA1C.

- Functional flexibility will be assessed by means of the timed sit and stand test. ${ }^{44}$

- Muscle strength will be measured using maximal isometric handgrip strength ${ }^{45} 46$ and isometric and isokinetic quadriceps strength and endurance. ${ }^{47}$

\section{Psychosocial well-being and intervention effectiveness}

Participants will fill in a booklet of questionnaires which will be administered as an online survey on a portable PC or tablet during the assessment visits. Furthermore, patients randomised to the PATHway intervention will also fill in a few further questionnaires on the PATHway platform, since needed for the health behavioural change module. These instruments assess among others, health, 
Table 2 Tabulated summary of study schedule

\begin{tabular}{|c|c|c|c|c|}
\hline & $\begin{array}{l}\text { Enrolment } \\
\text { TO }\end{array}$ & $\begin{array}{l}\text { Baseline } \\
\text { T1 }\end{array}$ & $\begin{array}{l}\text { FU3m } \\
\text { T2 }\end{array}$ & $\begin{array}{l}\text { FU6m } \\
\text { T3 }\end{array}$ \\
\hline Eligibility screen & $\mathrm{x}$ & & & \\
\hline Informed consent & $x$ & & & \\
\hline Allocation & $\mathrm{X}$ & & & \\
\hline PATHway intervention & & $\hookleftarrow$ & 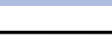 & $\longrightarrow$ \\
\hline Usual care (control) intervention & & $\longleftarrow$ & & $\rightarrow$ \\
\hline \multicolumn{5}{|l|}{ Assessments } \\
\hline Physical activity & & $\mathrm{x}$ & $x$ & $\mathrm{X}$ \\
\hline Demographic characteristics & $\mathrm{x}$ & & & $\mathrm{x}$ \\
\hline \multicolumn{5}{|l|}{ Health-related physical fitness } \\
\hline CPET & & $x$ & $x$ & $x$ \\
\hline Muscle strength and flexibility & & $\mathrm{X}$ & $x$ & $\mathrm{X}$ \\
\hline Quality of the vascular system & & $\mathrm{X}$ & $x$ & $\mathrm{X}$ \\
\hline Blood sampling & & $x$ & & $x$ \\
\hline Body composition & & $x$ & $x$ & $x$ \\
\hline \multicolumn{5}{|c|}{ Health-related QoL and psychosocial well-being } \\
\hline PHQ-9 & & $x$ & $x$ & $\mathrm{x}$ \\
\hline EQ-5D-5L questionnaire & & $\mathrm{x}$ & $x$ & $x$ \\
\hline SF-36 questionnaire & & $x$ & $x$ & $x$ \\
\hline Exercise self-efficacy scale & & $x$ & $x$ & $x$ \\
\hline Medication adherence & & $\mathrm{x}$ & $x$ & $\mathrm{X}$ \\
\hline Exercise barriers & & $\mathrm{x}$ & $x$ & $x$ \\
\hline Perceived stress scale & & $x$ & $x$ & $x$ \\
\hline ENRICHD & & $x$ & $x$ & $x$ \\
\hline Satisfaction/usability tests & & & & $\mathrm{X}$ \\
\hline Resource use data collection & & $x$ & $x$ & $x$ \\
\hline \multicolumn{5}{|l|}{ Safety monitoring } \\
\hline Three-day ECG Holter monitoring & & $x$ & $x$ & $\mathrm{x}$ \\
\hline Adverse event reporting & & $\diamond$ & 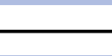 & $\longrightarrow$ \\
\hline \multicolumn{5}{|l|}{ Process evaluation (assessment of DSS) } \\
\hline $\begin{array}{l}\text { Validation of DSS outcomes captured in } \\
\text { reports by clinical experts }\end{array}$ & & $\mathrm{x}$ & $\mathrm{X}$ & $\mathrm{X}$ \\
\hline
\end{tabular}

Satisfaction/usability test will only be performed in the intervention group.

CPET, cardiopulmonary exercise test; DSS, decision support system; ENRICHD, Enhancing Recovery In Coronary Heart Disease; EQ-5D-5L, EuroQol-Five Dimensions-Five Levels Questionnaire; PHQ-9, Patient Health Questionnaire 9; QoL, Quality of Life; SF-36, Short Form 36; T, timepoint.

self-efficacy, well-being and quality of life. They will also collect data on the mechanisms hypothesised to mediate PATHway usage and maintenance of physical activity behaviour. An overview of the questionnaires used to assess outcome measures and guide risk factor behaviour change is shown in table 3. For an overview of the questionnaires administered at $\mathrm{t} 1, \mathrm{t} 2$ and $\mathrm{t} 3$ only, see table 2.

\section{Health economic evaluation}

The health economic evaluation will evaluate health outcomes and resource use consequences of the PATHway intervention. The analysis will be conducted from a societal perspective to include resource costs falling on the health service and on the individual, together with potential productivity benefits associated with return to work where applicable. HRQoL outcomes will be estimated in terms of Quality Adjusted Life Years (QALYs). These data will be generated using completed EuroQol-Five Dimensions-Five Levels Questionnaires. ${ }^{48}$ The cost of delivering the programme, the health service costs, the costs falling on the participants and the productivity effects in terms of participant's employment will be calculated. Information on participants' resource use will be obtained by a researcher by means of an interviewer-administered 





Table 4 Overview of main categories for health economics assessment

\section{Direct healthcare costs}

Concomitant medications

Visits to healthcare

professionals

Outpatient visits

ER visits

Inpatient admissions

\section{Direct costs to the patient/carer}

Travel costs

Home adaptations

\section{Productivity costs to patient/carer}

Time away from

employment/main activity

\section{Quality of Life}

Quality of life

EuroQol-Five Dimensions-

Five Levels Questionnaire

questionnaire at $\mathrm{t} 1, \mathrm{t} 2$ and $\mathrm{t} 3$ as shown in table 4 . The incremental cost-effectiveness ratio will be calculated as the cost per QALY gained by using the intervention.

Observed results will be extrapolated beyond the 6-month intervention period in the clinical trial, in order to explore the potential lifetime cost-effectiveness of PATHway. Statistical and decision-modelling methods will be used to undertake the extrapolation, which will apply to costs as well as effects. The model will link changes in exercise capacity and cardiovascular risk profiles to recurrent cardiovascular events and CVD mortality in order to estimate the cost-per-QALY associated with the PATHway programme. The post-trial extrapolation for each arm uses estimates of long-term recurrence from the literature and the observed recurrence rates within the period of the trial, accounting for any differences in resource use, life expectancy and quality of life.

\section{Safety monitoring}

All serious adverse events (SAE) will be reported continuously to a Data Safety and Monitoring Committee composed of four cardiologists. SAE are defined as all-cause mortality, hospitalisation for CVD or serious atrial or ventricular arrhythmia. Other adverse events will include training-related adverse events such as muscle, tendon or joint problems that will preclude exercise participation or other diseases that require an interruption of the exercise intervention. Participants will also wear a 3-lead ECG Zensor Device (Intelesens, Belfast, North Ireland) monitoring system for three consecutive days at the three time points of measurement. The participants will be asked to wear the Zensor simultaneously with the ActiGraph device.

\section{Qualitative outcomes}

The PATHway system one-on-one interviews guided by the Health IT Usability Evaluation Model (Health-ITUEM), ${ }^{49}$ will be used to evaluate both subjective (perceived ease of use and perceived usefulness) and objective (efficiency and effectiveness) measures as part of the PATHway debrief, with participants and stakeholders (ie, physiotherapists, cardiologists, cardiac nurses). Face-to-face interviews will be conducted by a trained interviewer to the point of data saturation. Participants will be asked about their opinions on the PATHway programme, especially in relation to perceptions of improved self-management and confidence in DSSs; the influence of peer mentors; the programme language; the level of content remembered as well as perceptions of intrusiveness, timing and content. All interviews will be semistructured in nature to allow the extrapolation of novel patient-led topics in relation to the intervention. All interviews will be audio-taped and transcribed verbatim and analysed using Braun and Clarke's thematic analysis five-step framework. ${ }^{50}$ Audit trails will be used throughout this analysis to ensure transparency throughout the development process from raw interview transcripts to themes to user feedback formulations to the technology team.

\section{Data management and analysis}

Each randomised participant will be assigned a PIC. This unique identification number will be used on all case report forms, electronic databases and within the PATHway platform. Data will be recorded in hardcopy at the time of the measurement and will subsequently be entered electronically in 'RedCap', an open source clinical trial software for electronic data capture and data management. The database will be hosted and secured hardcopies will be stored in a secured filing cabinet at the participating sites. The data entry screens in RedCap will resemble the hard copy case report forms approved by the ethical committee. Checks will be automatically applied when entering the data in the database based on preset ranges. Missing data will also be automatically detected and data query reports will be sent to the local data manager. The type of activity that an individual user may undertake is regulated by the privileges associated with his/her user identification code and passwords. After submission of the data, researchers responsible at the participating sites cannot make any changes. Appropriate changes can only be made by the principle data manager.

All data analyses and reporting will be performed according to best practice and reported in agreement with Consolidated Standards of Reporting Trials guidelines and the Consolidated criteria for reporting qualitative research. ${ }^{51-53}$ Data analysis will be performed using SAS version 9.4. Baseline demographic characteristics and parameters related to pathology will be reported descriptively and will in first instance be compared across countries and between treatment groups to assess whether further analyses need adjustment for any unbalanced variables. In addition, these basic demographic characteristics will be compared between non-participating eligible participants and eligible participants enrolled in the trial to evaluate the potential of selection bias. All 
primary and secondary outcome parameters as previously described will be analysed on an intention-to-treat principle according to initial randomisation and using data collected at 6 months following completion of the phase 2 CR programme. To account for missing data, we will impute the missing value with the last measured value. The Shapiro-Wilk test will be applied to assess normality. The mixed linear models will be used to assess differences between groups. All tests will be two-sided and a $p$ value $<0.05$ will be considered statistically significant.

\section{ETHICS AND DISSEMINATION}

The study will be conducted in accordance with the principles stated in the Declaration of Helsinki. ${ }^{54}$ Participants will only be enrolled in the study after signing written informed consent. None of the principal investigators in the PATHway study have any financial interest to declare. This protocol and the template informed consent have been reviewed and approved by the director and clinical director of the PATHway study and are approved by the ethical committee of the UZ Leuven/ KU Leuven and the Research Ethics Committee of both Irish hospital partners (KU Leuven: S59023; Mater Misericordiae Hospital Dublin: 1/378/1846; Beaumont Hospital Dublin: 16/50), as well as Dublin City University (DCU: REC2016/123) with respect to scientific content and compliance with applicable research and human subjects regulations. In case of any amendments to the consent form during the study, the volunteers will be given a copy of the updated form and any written information. We will disseminate the PATHway study results via peer-reviewed scientific journals and presentations at local, national and international congresses and events.

\section{CONCLUSION}

It has recently been suggested that a comprehensive telerehabilitation programme for participants with cardiac disease should include telemonitoring, telecoaching, social networking and e-learning in order to achieve significant long-term effects on the participants' health condition. ${ }^{18}$ To date, most of the telerehabilitation programmes have, however, only included one or two of these components. Furthermore, although exercise and physical activity are key in a CR programme, current guidelines clearly state that a CR programme should involve all of the core components. ${ }^{10}$ To the best of our knowledge, PATHway is the first system that provides such a comprehensive cardiac telerehabilitation programme which is fully patient-tailored based on personal needs and preferences. More specific, participants will be provided with a personalised exercise prescription which will be formulated based on the clinical status of the participants and also taking personal preferences and exercise goals into account. In addition to this and based on an evaluation of other lifestyle behaviours, participants will be encouraged to set personalised goals for adopting a healthy lifestyle. An e-learning environment including written information and videos focusing on the different core components of secondary prevention programmes will help participants to get a better understanding of their disease and its management. It should be noted that PATHway was not designed to replace current supervised phase $2 \mathrm{CR}$ programmes, but it aims to offer an alternative for those patients who cannot complete a phase 2 CR programme due to work or other commitments and it is intended to support a physically active and healthy lifestyle beyond the phase 2 programme. The main goal of this pilot trial is to gain insight into the change in health status of the patient and the efficacy of the system. This will allow further dissemination of the PATHway platform in the future.

\section{LIMITATIONS}

As the study is of relatively short duration, no data will be available beyond 6 months. Furthermore, the study is underpowered to detect statistically significant changes for a majority of secondary outcomes. However, it might be anticipated that when we are able to demonstrate an increased uptake of physical activity 6 months following completion of the ambulatory phase 2 CR programme by using PATHway, this will result in an enhancement of cardiovascular health in the longer term. Hence, this study would then provide justification for prospective trials to evaluate the longer-term effectiveness on cardiovascular health and the cost-effectiveness of the PATHway platform in this population and also other chronic disease populations.

\section{Expected outcomes and future contributions}

At this stage, PATHway aims to support patients with cardiac disease in maintaining a physically active lifestyle and where possible to further increase their levels of physical activity. In addition, it will help participants to choose a healthy lifestyle. If the results of PATHway are positive, the PATHway platform can be extended to other patient populations with chronic disease including patients with peripheral artery disease, chronic stroke, chronic obstructive lung disease and also in the primary prevention of CVD in persons with established cardiovascular risk factors for whom exercise and the adoption of a healthy lifestyle is a core component in their disease management.

\section{Author affiliations}

${ }^{1}$ Department of Cardiovascular Sciences, KU Leuven, Leuven, Belgium ${ }^{2}$ Department of Physical Education and Sport Sciences, University of Limerick, Limerick, Ireland

${ }^{3}$ University of Glasgow, Institute of Health and Wellbeing, Glasgow, Scotland ${ }^{4}$ Department of Health \& Human Performance, Dublin City University, Dublin, Ireland ${ }^{5}$ Centre for Research and Technology, Institute of Applied Biosciences, Hellas, Greece

${ }^{6}$ Division of Cardiology, University Hospitals Leuven, Leuven, Belgium ${ }^{7}$ Department of Rehabilitation Sciences, KU Leuven, Leuven, Belgium

Contributors JC, RB and VAC drafted the manuscript. KM is the principal coordinator of the PATHway project. All other authors revised the manuscript and provided input in their area of expertise: $A B, C G$ and MA for health economics; NM, NMc and WB for physiological measurements; CW and DW considering psychological components; IC, DF, AT and NMa for the technical aspects of the 
PATHway system. The PATHway project was conceived by the PATHway consortium (http://www.pathway2health.eu/).

Funding This work was supported by the European Union's Horizon 2020 Framework Programme for Research and Innovation Action under Grant Agreement no. 643491. PATHway: Technology enabled behavioural change as a pathway towards better self-management of CVD (www.pathway2health.eu).

Competing interests None declared.

Ethics approval This protocol and the template informed consent have been reviewed and approved by the director and clinical director of the PATHway study and are approved by the ethical committee of the UZ Leuven/ KU Leuven and the Research Ethics Committee of both Irish hospital partners (KU Leuven: S59023; Mater Misericordiae Hospital Dublin: 1/378/1846; Beaumont Hospital Dublin: 16/50), as well as Dublin City University (DCU: REC2016/123).

Provenance and peer review Not commissioned; externally peer reviewed.

Data sharing statement All available data are included in this manuscript as text, figures, tables and a checklist. Please contact the corresponding author in case of any additional requirements.

Open Access This is an Open Access article distributed in accordance with the Creative Commons Attribution Non Commercial (CC BY-NC 4.0) license, which permits others to distribute, remix, adapt, build upon this work non-commercially, and license their derivative works on different terms, provided the original work is properly cited and the use is non-commercial. See: http://creativecommons.org/ licenses/by-nc/4.0/

(C) Article author(s) (or their employer(s) unless otherwise stated in the text of the article) 2017. All rights reserved. No commercial use is permitted unless otherwise expressly granted.

\section{REFERENCES}

1. Vanhees L, Rauch B, Piepoli M, et al. Importance of characteristics and modalities of physical activity and exercise in the management of cardiovascular health in individuals with cardiovascular disease (Part III). Eur J Prev Cardiol 2012;19:1333-56.

2. Cardiology EHNaESo. European Cardiovascular Disease Statistics. 2012. http://www.ehnheart.org/cvd-statistics/cvd-statistics-2012. html

3. Townsend N, Nichols M, Scarborough P, et al. Cardiovascular disease in Europe--epidemiological update 2015. Eur Heart J 2015;36:2696-705.

4. Taylor RS, Brown A, Ebrahim S, et al. Exercise-based rehabilitation for patients with coronary heart disease: systematic review and meta-analysis of randomized controlled trials. Am J Med 2004;116:682-92.

5. Oldridge $\mathrm{N}$. Exercise-based cardiac rehabilitation in patients with coronary heart disease: meta-analysis outcomes revisited. Future Cardiol 2012;8:729-51.

6. Arbit B, Azarbal B, Hayes SW, et al. Prognostic contribution of Exercise Capacity, Heart Rate Recovery, Chronotropic Incompetence, and myocardial perfusion Single-Photon Emission Computerized Tomography in the prediction of cardiac death and All-Cause Mortality. Am J Cardiol 2015;116:1678-84.

7. Vanhees L, Fagard R, Thijs L, et al. Prognostic significance of peak exercise capacity in patients with coronary artery disease. J Am Coll Cardiol 1994;23:358-63.

8. Keteyian SJ, Brawner CA, Savage PD, et al. Peak aerobic capacity predicts prognosis in patients with coronary heart disease. Am Heart J 2008;156:292-300.

9. Vanhees L, Stevens A, Schepers D, et al. Determinants of the effects of physical training and of the complications requiring resuscitation during exercise in patients with cardiovascular disease. Eur $J$ Cardiovasc Prev Rehabil 2004;11:304-12.

10. Piepoli MF, Corrà U, Adamopoulos S, et al. Secondary prevention in the clinical management of patients with cardiovascular diseases. core components, standards and outcome measures for referral and delivery: a policy statement from the cardiac rehabilitation section of the European Association for Cardiovascular Prevention \& Rehabilitation. endorsed by the Committee for Practice guidelines of the european Society of Cardiology. Eur J Prev Cardiol 2014;21:664--81.

11. Bjarnason-Wehrens B, McGee H, Zwisler AD, et al. Cardiac rehabilitation in Europe: results from the european cardiac Rehabilitation Inventory Survey. Eur J Cardiovasc Prev Rehabil 2010;17:410-8.
12. Dunlay SM, Witt BJ, Allison TG, et al. Barriers to participation in cardiac rehabilitation. Am Heart J 2009;158:852-9.

13. Artinian NT, Fletcher GF, Mozaffarian D, et al. Interventions to promote physical activity and dietary lifestyle changes for cardiovascular risk factor reduction in adults: a scientific statement from the American Heart Association. Circulation 2010;122:406-41.

14. Michie S, van Stralen MM, West R. The behaviour change wheel: a new method for characterising and designing behaviour change interventions. Implement Sci 2011;6:42

15. Perk J, De Backer G, Gohlke H, et al. European guidelines on cardiovascular disease prevention in clinical practice (version 2012). The fifth Joint Task Force of the European Society of Cardiology and other societies on Cardiovascular Disease Prevention in clinical practice (constituted by representatives of nine societies and by invited experts). Eur Heart J 2012;33:1635.

16. Crookham J. A guide to exercise prescription. Prim Care 2013;40:801-20.

17. Williams SL, French DP. What are the most effective intervention techniques for changing physical activity self-efficacy and physical activity behaviour--and are they the same? Health Educ Res 2011;26:308-22.

18. Frederix I, Vanhees L, Dendale P, et al. A review of telerehabilitation for cardiac patients. J Telemed Telecare 2015;21:45-53.

19. Franklin NC, Lavie CJ, Arena RA. Personal health technology: a new era in cardiovascular disease prevention. Postgrad Med 2015;127:150-8.

20. Buys R, Claes J, Walsh D, et al. Cardiac patients show high interest in technology enabled cardiovascular rehabilitation. BMC Med Inform Decis Mak 2016;16:95

21. Chan AW, Tetzlaff JM, Altman DG, et al. SPIRIT 2013 statement: defining standard protocol items for clinical trials. Ann Intern Med 2013;158:200-7.

22. Chan AW, Tetzlaff JM, Gøtzsche PC, et al. SPIRIT 2013 explanation and elaboration: guidance for protocols of clinical trials. BMJ 2013;346:e7586.

23. Perk J, De Backer G, Gohlke H, et al. European guidelines on cardiovascular disease prevention in clinical practice (version 2012): The fifth Joint Task Force of the European Society of Cardiology and other societies on Cardiovascular Disease Prevention in clinical practice (constituted by representatives of nine societies and by invited experts). Atherosclerosis 2012;223:1-68.

24. Heidbüchel $H$, Corrado D, Biffi $A$, et al. Recommendations for participation in leisure-time physical activity and competitive sports of patients with arrhythmias and potentially arrhythmogenic conditions. Part II: ventricular arrhythmias, channelopathies and implantable defibrillators. Eur J Cardiovasc Prev Rehabil 2006;13:676-86.

25. Heidbüchel H, Panhuyzen-Goedkoop N, Corrado D, et al. Recommendations for participation in leisure-time physical activity and competitive sports in patients with arrhythmias and potentially arrhythmogenic conditions part I: supraventricular arrhythmias and pacemakers. Eur J Cardiovasc Prev Rehabil 2006;13:475-84.

26. Pelliccia A, Corrado D, Bjørnstad HH, et al. Recommendations for participation in competitive sport and leisure-time physical activity in individuals with cardiomyopathies, myocarditis and pericarditis. Eur J Cardiovasc Prev Rehabil 2006;13:876-85.

27. Giannuzzi P, Saner H, Björnstad H, et al. Secondary prevention through cardiac rehabilitation: position paper of the Working Group on Cardiac Rehabilitation and Exercise Physiology of the European Society of Cardiology. Eur Heart J 2003;24:1273-8.

28. Börjesson M, Assanelli D, Carré F, et al. ESC Study Group of Sports Cardiology: recommendations for participation in leisure-time physical activity and competitive sports for patients with ischaemic heart disease. Eur J Cardiovasc Prev Rehabil 2006;13:137-49.

29. Budts W, Börjesson M, Chessa M, et al. Physical activity in adolescents and adults with congenital heart defects: individualized exercise prescription. Eur Heart J 2013;34-3669-74.

30. Mellwig KP, van Buuren F, Gohlke-Baerwolf C, et al. Recommendations for the management of individuals with acquired valvular heart diseases who are involved in leisure-time physical activities or competitive sports. Eur J Cardiovasc Prev Rehabil 2008;15:95-103.

31. Microsoft. Microsoft Band. 2016.

32. Filos D, Triantafyllidis A, Chouvarda I, et al. PATHway: decision support in Exercise Programmes for Cardiac Rehabilitation. Stud Health Technol Inform 2016;224:40-5.

33. Mezzani A, Hamm LF, Jones AM, et al. Aerobic exercise intensity assessment and prescription in cardiac rehabilitation: a joint position statement of the European Association for Cardiovascular Prevention and Rehabilitation, the American Association of Cardiovascular and Pulmonary Rehabilitation and the Canadian 
Association of cardiac Rehabilitation. Eur J Prev Cardiol 2013;20:442-67.

34. Middelweerd A, Mollee JS, van der Wal CN, et al. Apps to promote physical activity among adults: a review and content analysis. Int $J$ Behav Nutr Phys Act 2014;11:97.

35. Walsh DMK, Buys R, Claes J, et al. The development and co-design of the PATHway intervention: a theory-driven eHealth platform for the self-management of cardiovascular disease. Unpublished data.

36. Kahn EB, Ramsey LT, Brownson RC, et al. The effectiveness of interventions to increase physical activity. A systematic review. Am J Prev Med 2002;22:73-107.

37. Consortium P. PATHway platform. 2016.

38. Montalescot G, Sechtem U, Achenbach S, et al. 2013 ESC guidelines on the management of stable coronary artery disease: the Task Force on the management of stable coronary artery disease of the European Society of Cardiology. Eur Heart J 2013;34:2949.

39. Henderson RA, O'Flynn N; Guideline Development Group. Management of stable angina: summary of NICE guidance. Heart 2012;98:500-7.

40. Mezzani A, Agostoni P, Cohen-Solal A, et al. Standards for the use of cardiopulmonary exercise testing for the functional evaluation of cardiac patients: a report from the Exercise Physiology Section of the European Association for Cardiovascular Prevention and Rehabilitation. Eur J Cardiovasc Prev Rehabil 2009;16:249-67.

41. ACSM. ACSM's Health-related physical fitness assessment manual, 2013.

42. Onkelinx S, Cornelissen V, Goetschalckx K, et al. Reproducibility of different methods to measure the endothelial function. Vasc Med 2012;17-79-84.

43. Corretti MC, Anderson TJ, Benjamin EJ, et al. Guidelines for the ultrasound assessment of endothelial-dependent flow-mediated vasodilation of the brachial artery: a report of the International Brachial Artery Reactivity Task Force. J Am Coll Cardiol 2002;39:257-65.
44. Jones CJ, Rikli RE, Beam WC. A 30-s chair-stand test as a measure of lower body strength in community-residing older adults. Res $Q$ Exerc Sport 1999;70:113-9.

45. Ribom EL, Mellström D, Ljunggren Ö, et al. Population-based reference values of handgrip strength and functional tests of muscle strength and balance in men aged 70-80 years. Arch Gerontol Geriatr 2011;53:e114-e117.

46. Roberts HC, Denison HJ, Martin HJ, et al. A review of the measurement of grip strength in clinical and epidemiological studies: towards a standardised approach. Age Ageing 2011;40:423-9.

47. Thomaes T, Thomis M, Onkelinx S, et al. Muscular strength and diameter as determinants of aerobic power and aerobic power response to exercise training in CAD patients. Acta Cardiol 2012;67:399-406.

48. EuroQol Group. EuroQol--a new facility for the measurement of healthrelated quality of life. Health Policy 1990;16:199-208.

49. Brown W, Yen PY, Rojas M, et al. Assessment of the Health IT Usability evaluation Model (Health-ITUEM) for evaluating mobile health (mHealth) technology. J Biomed Inform 2013;46:1080-7.

50. Braun V, Clarke V. Using thematic analysis in psychology. Qual Res Psychol 2006;3:77-101.

51. Moher D, Hopewell S, Schulz KF, et al. CONSORT 2010 explanation and elaboration: updated guidelines for reporting parallel group randomised trials. Int J Surg 2012;10:28-55.

52. Schulz KF, Altman DG, Consort MD. Statement: updated guidelines for reporting parallel group randomised trials. Int J Surg 2010;2011;9:672-7.

53. Tong A, Sainsbury P, Craig J. Consolidated criteria for reporting qualitative research (COREQ): a 32-item checklist for interviews and focus groups. Int J Qual Health Care 2007;19:349-57.

54. Williams J. The declaration of Helsinki and public health. Bull World Health Organ 2008;86:650-1. 